\title{
Two cases - Orthostatic hypotension following retroperitoneal lymph node dissection
}

Diana E. Magee, MD, MPH; Robert J. Hamilton, MD, MPH

Division of Urology, Department of Surgery, University Health Network, University of Toronto

Cite as: Can Urol Assoc J 2020 March 30; Epub ahead of print.

http://dx.doi.org/10.5489/cuaj.6419

Published online March 30, 2020

$* * *$

\section{Introduction}

Retroperitoneal lymph node dissection (RPLND) is an integral part of the management of testicular cancer ${ }^{1}$ and an adjunct to the treatment of other advanced intra-abdominal malignancies. ${ }^{2-5}$ It is, however, associated with a significant risk of complications particularly in the post-chemotherapy setting. ${ }^{6}$ In the following two cases, we report the development of significant orthostatic hypotension following RPLND, a rarely described postoperative complication.

\section{Case reports}

\section{Patient one}

A 23-year-old male presented with right sided flank pain and gross hematuria. Computed tomography (CT) of the abdomen, pelvis and thorax revealed a large right renal mass, significant retroperitoneal nodal disease and small bilateral pulmonary metastases (Figure 1). Renal biopsy revealed a poorly differentiated neuroendocrine carcinoma.

He was treated with six cycles of Etoposide and Cisplatin. His post-chemotherapy imaging showed interval improvement in the renal mass and retroperitoneal nodal disease with resolution of thoracic lesions. His case was discussed at multidisciplinary tumour boards and due to his young age, overall fitness and patient desire it was felt appropriate to consider surgical consolidation of his abdominal disease with a radical nephrectomy and RPLND.

An adrenal sparing right radical nephrectomy with extended template RPLND was completed. Grossly identifiable disease was visualized in the para, retro and interaortocaval spaces as well as the para and retroaortic spaces. All the post-ganglionic sympathetic fibres were sacrificed bilaterally due to their involvement in the masses except for one trunk at the L3 level on the right which was grafted using the genito-femoral nerve to bridge to the hypogastric plexus.

His post-operative recovery was uneventful. He met appropriate milestones and was discharged home on post-operative day four in stable condition. Unfortunately, he returned on 
post-operative day eight with progressively worsening postural dizziness. He was found to have profound postural hypotension and reflex tachycardia. With standing, his blood pressure dropped to $65 / 45$ with a compensatory heart rate of 166 . Supine vitals were normal. Routine bloodwork was also normal.

He was admitted to the hospital for support and further work-up. Dehydration and adrenal insufficiency were ruled out. Internal Medicine and Neurology were consulted. They felt this presentation represented orthostatic hypotension from sympathetic injury and recommended compression stockings, increased salt intake and the initiation of Fludrocortisone $0.1 \mathrm{mg}$ orally once daily. Due to minimal improvement, Midodrine $5 \mathrm{mg}$ orally twice daily as needed was added. With this addition, his blood pressure and heart rate with standing improved to 106/70 and 140, respectively. He remained mildly symptomatic with these postural changes but much improved compared to presentation and was discharged with compression stockings, Fludrocortisone and Midodrine. At six months follow up he had been taken off Fludrocortisone and Midodrine and switched to a beta-blocker for rate control. By one year post-operatively he no longer required medications and was participating in active sports including soccer.

\section{Patient two}

A 28-year-old patient presented with a one-month history of a left sided testicular mass. Investigations revealed a left sided testicular tumour with significant abdominal lymphadenopathy (Figure 2). He underwent a radical orchiectomy which revealed a pT1 seminoma. However, his post-operative AFP continued to rise, suggestive of a discordance between his testicular pathology and overall disease type. Therefore, he was treated as a nonseminoma and underwent four cycles of Bleomycin, Etoposide and Cisplatin.

Post-chemotherapy, with negative markers, his imaging showed persistent retroperitoneal and retrocrural disease. Therefore, the decision was made to pursue a RPLND and retrocrural dissection. Intra-operatively there was intense desmoplastic reaction in the retroperitoneum which made dissection of the masses challenging. The retrocrural mass was resected in conjunction with Thoracic Surgery by gaining access to the lesser sac and dividing the crus. The sympathetic chains were resected bilaterally due to their adherence to the lymphatic tissue and lack of dissectible plane between the structures.

After an uneventful post-operative course, he was discharged home on post-operative day nine in stable condition. However, on post-operative day 12 he presented to the emergency department with postural related pre-syncopal symptoms. His blood pressure with sitting was $68 / 36$ with a heart rate of 150 . Routine blood work and cortisol were within normal limits.

The Internal Medicine service was consulted. They felt his orthostatic hypotension was likely secondary to autonomic insufficiency due to surgical resection. Recommendations included liberal fluid and salt intake, Fludrocortisone $0.2 \mathrm{mg}$ orally once daily and compression stockings. Due to minimal improvement, Midodrine added to regimen starting at $2.5 \mathrm{mg}$ orally three times daily and titrated up to $5 \mathrm{mg}$ three times daily. The patient was discharged home on 
Fludrocortisone and Midodrine using compression stockings during the day. At the time of discharge his blood pressure was 123/78 with a heart rate of 107 on standing. Approximately three months post-operatively he had recovered and was able to function normally aside from an occasional episode of dizziness and tachycardia.

\section{Discussion}

These two patients, separated by years and over 150 consecutive RPLNDs at our institution, developed significant orthostatic hypotension secondary to sympathetic disruption requiring inpatient multidisciplinary management and extended pharmaceutical support. A previous case series which described post-RPLND complications reported one occurrence of orthostatic hypotension but to our knowledge this is the first case series describing in detail the specifics surrounding its development and subsequent management. ${ }^{7}$

We have thought critically about the anatomy of dissection of these two cases to understand why they developed this complication, while hundreds of other, often more extensive RPLNDs did not lead to this complication. We have concluded that it is the extent of sympathetic denervation, in particular the suprahilar dissection that lead to this postural hypotension. The key sympathetic anatomy to consider is as follows: a) post-ganglionic sympathetic fibres from L2-L4 that connect to the hypogastric plexus; b) post-ganglionic sympathetic fibres that travel to the inferior mesenteric ganglion, alongside the inferior mesenteric artery and out to the large bowel; c) sympathetic chains; d) sympathetic fibres coalescing at the renal ganglia, aorticorenal ganglia and the superior mesenteric ganglia. Resecting any individual element is not enough to cause hypotension, but if all or nearly all elements are resected, postural hypotension is more likely. The most critical, we feel, are the superior mesenteric ganglia and fibres heading to the small bowel alongside the superior mesenteric artery (SMA). The ability to suddenly increase venous return from the region of bowel supplied by the SMA is key to maintaining upright posture.

As there are no definitive guidelines or significant body of literature on the subject, we found the following steps in the management of neurogenic orthostatic hypotension helpful in the treatment of our patients: rule out adrenal insufficiency, consultation with internal medicine, intravenous fluids, high salt diet, compression stockings, abdominal binder, medical therapy (fludricortisone, midodrine, erythropoietin), and rehabilitation. Combination medical therapy is effective but needs to be tailored to the individual patient and drug response. ${ }^{8}$ Patients can be counselled that symptoms likely will improve over time but may not fully return to pre-operative normal state.

The development of orthostatic hypotension following RPLND represents a rare complication and a significant source of patient morbidity. While it is a difficult treatment problem, the use of a multidisciplinary team with implementation of treatments in order of increasing aggressiveness can lead to the return of normal daily functioning for affected patients. 


\section{References}

1. Albers P, Albrecht W, Algaba F, et al. Guidelines on Testicular Cancer: 2015 Update. Eur Urol. 2015;68(6):1054-1068.

2. Whitson JM, Harris CR, Reese AC, Meng MV. Lymphadenectomy improves survival of patients with renal cell carcinoma and nodal metastases. J Urol. 2011;185(5):1615-1620.

3. Capitanio U, Matloob R, Suardi N, et al. The extent of lymphadenectomy does affect cancer specific survival in pathologically confirmed T4 renal cell carcinoma. Urologia. 2012;79(2):109-115.

4. Bochner BH. Lymph Node Dissection for Advanced Bladder Cancer: Is There a Role? Eur Urol Focus. 2019.

5. Reibetanz J, Rinn B, Kunz AS, et al. Patterns of Lymph Node Recurrence in Adrenocortical Carcinoma: Possible Implications for Primary Surgical Treatment. Ann Surg Oncol. 2019;26(2):531-538.

6. Baniel J, Sella A. Complications of retroperitoneal lymph node dissection in testicular cancer: primary and post-chemotherapy. Semin Surg Oncol. 1999;17(4):263-267.

7. Tanaka T, Kitamura H, Kunishima Y, et al. Modified and bilateral retroperitoneal lymph node dissection for testicular cancer: peri- and postoperative complications and therapeutic outcome. Jpn J Clin Oncol. 2006;36(6):381-386.

8. Robertson D, Davis TL. Recent advances in the treatment of orthostatic hypotension. Neurology. 1995;45(4 Suppl 5):S26-32.

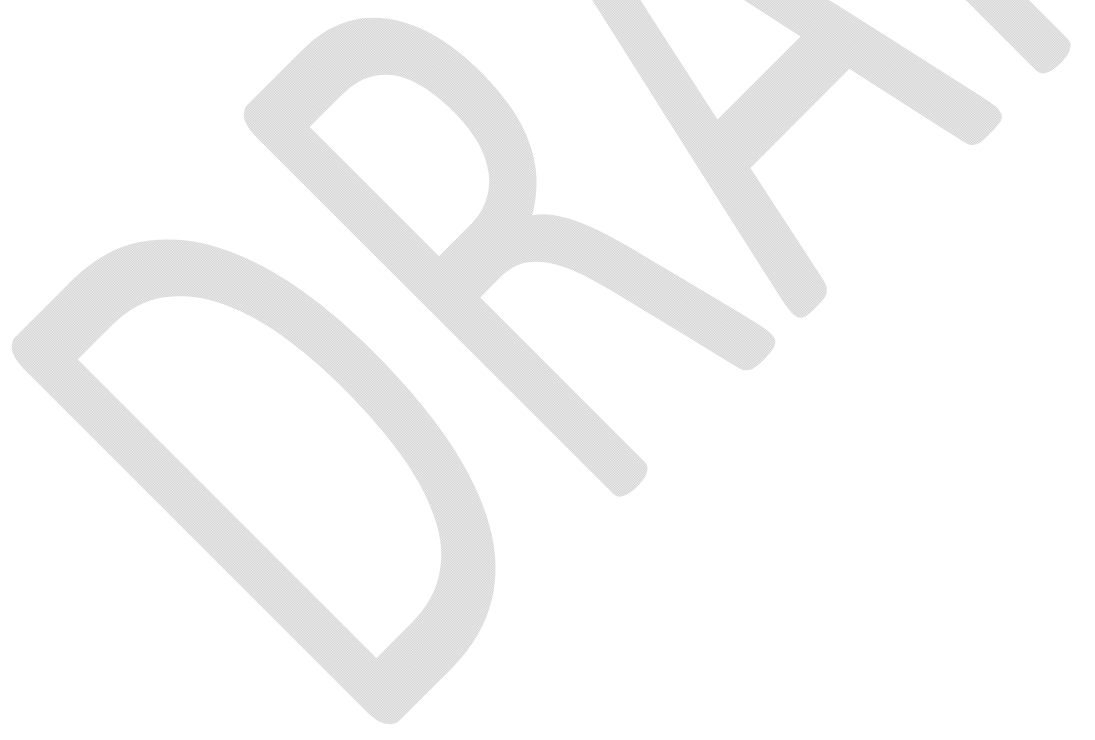


Fig. 1. Contrast enhanced (venous phase) computed tomography scan in the pre-chemotherapy setting demonstrating: (A) the superior extension of nodal deposits and the significant right renal mass in the coronal plane; $(\boldsymbol{B})$ the burden of disease in a representative axial section.

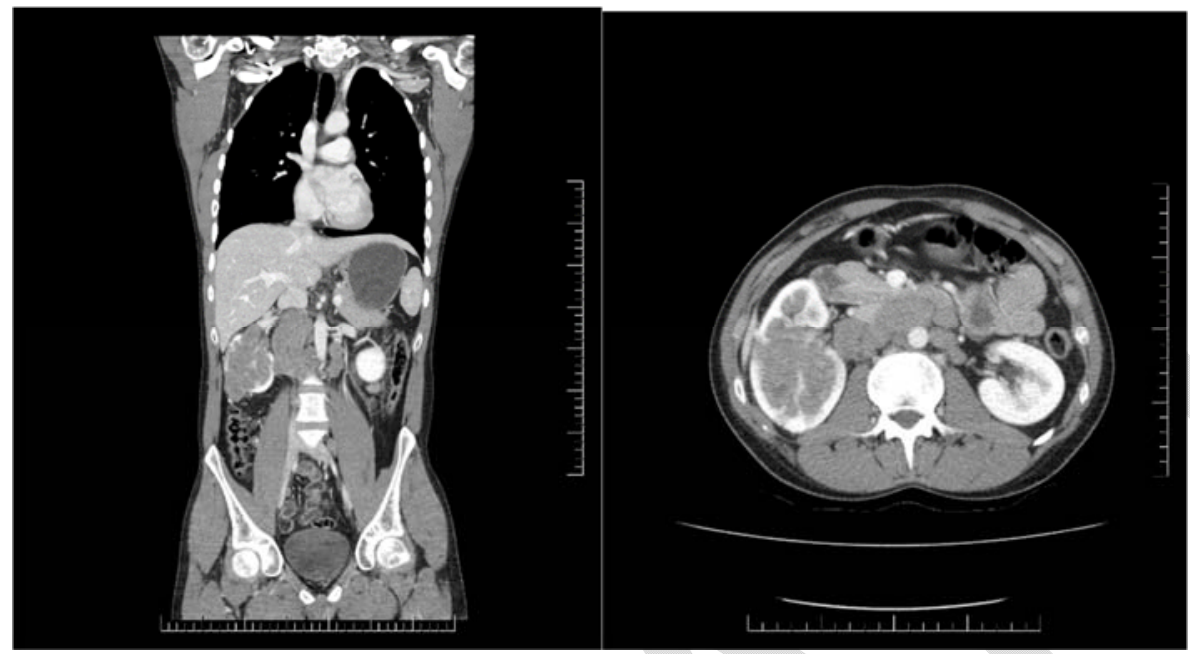

Fig. 2. Contrast enhanced (venous phase) computed tomography scan in the pre-chemotherapy setting demonstrating: (A) the superior extension of nodal deposits into the retrocrural space in the coronal plane; $(\boldsymbol{B})$ the burden of disease in a representative axial section.
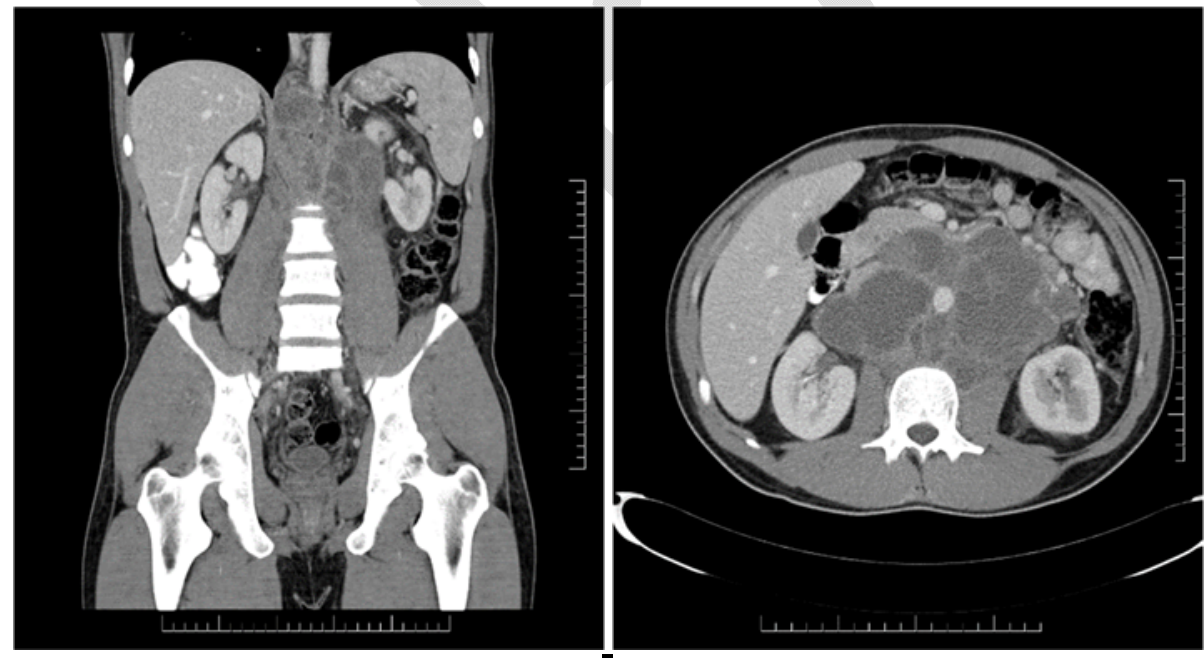